\title{
Direct comparisons of compressible magnetohydrodynamics and reduced magnetohydrodynamics turbulence
}

\author{
Pablo Dmitruk and William H. Matthaeus \\ Bartol Research Institute, University of Delaware, Newark, Delaware 19716 \\ Sean Oughton \\ Department of Mathematics, University of Waikato, Private Bag 3105, Hamilton, New Zealand
}

(Received 13 July 2005; accepted 5 October 2005; published online 11 November 2005)

\begin{abstract}
Direct numerical simulations of low Mach number compressible three-dimensional magnetohydrodynamic (CMHD3D) turbulence in the presence of a strong mean magnetic field are compared with simulations of reduced magnetohydrodynamics (RMHD). Periodic boundary conditions in the three spatial coordinates are considered. Different sets of initial conditions are chosen to explore the applicability of RMHD and to study how close the solution remains to the full compressible MHD solution as both freely evolve in time. In a first set, the initial state is prepared to satisfy the conditions assumed in the derivation of RMHD, namely, a strong mean magnetic field and plane-polarized fluctuations, varying weakly along the mean magnetic field. In those circumstances, simulations show that RMHD and CMHD3D evolve almost indistinguishably from one another. When some of the conditions are relaxed the agreement worsens but RMHD remains fairly close to CMHD3D, especially when the mean magnetic field is large enough. Moreover, the well-known spectral anisotropy effect promotes the dynamical attainment of the conditions for RMHD applicability. Global quantities (mean energies, mean-square current, and vorticity) and energy spectra from the two solutions are compared and point-to-point separation estimations are computed. The specific results shown here give support to the use of RMHD as a valid approximation of compressible MHD with a mean magnetic field under certain but quite practical conditions. (C) 2005 American Institute of Physics. [DOI: 10.1063/1.2128573]
\end{abstract}

\section{INTRODUCTION}

In the presence of a strong or moderately strong largescale mean magnetic field, the equations of compressible magnetohydrodynamics can be approximated, under certain conditions, by the so-called equations of reduced magnetohydrodynamics (RMHD). In RMHD a subset of the nonlinear couplings of full MHD are retained. The physical picture is that of two-dimensional MHD fluctuations in planes perpendicular to the mean magnetic field, which are coupled by long wavelength modes (the Alfvén waves) along the meanfield direction.

Original derivations of the RMHD equations ${ }^{1-3}$ need to assume two conditions: (i) that fluctuations are small as compared to the mean magnetic field and (ii) that gradients of the fluctuations along the direction of the mean magnetic field are much smaller than gradients in the perpendicular direction. A third issue is the role of component fluctuations parallel to the mean field. When deriving RMHD from incompressible equations, ${ }^{2}$ the parallel fluctuations obey a passive equation that is added to the RMHD system. However, starting from a compressible plasma derivation, ${ }^{4}$ the parallel fluctuations are required to be small in establishing RMHD.

In a formal asymptotic derivation, ${ }^{4}$ RMHD is obtained from the compressible MHD equations at lower Mach number, for the strong mean magnetic-field case [condition (i)] and large aspect ratio [i.e., condition (ii)]. High-frequency modes are eliminated in this procedure, and RMHD emerges as a suitable description of low-frequency magnetohydrody- namics. Two limits are involved-low Mach number and strong magnetic field - so that the collapse in dimensionality and the approach to incompressibility become intertwined in establishing RMHD. More elaborate derivations of RMHD equations also include complications due to effects of weak spatial inhomogeneity and/or toroidal geometry., 5

The practical advantages of using the RMHD equations are clear. First (see below), the dynamics can be fully described in terms of two scalar fields as compared to the seven scalar fields needed for the full MHD equations. Second, as some of the couplings are not included, fewer nonlinear terms (often the most demanding terms in numerical simulations) need to be computed. Third, since fast compressible modes are eliminated at the onset, lower spatial and time resolutions are usually required. Finally, in situations where a large aspect ratio of the geometrical domain already exists, use of the full MHD equations with equal number of modes in all directions would be computationally prohibitive, while the inherent anisotropic property of RMHD is fully consistent with this geometry and computationally feasible. As a result of these clear advantages, RMHD has been widely employed in applications where a large mean magnetic field and a large aspect ratio exist. Included in those cases are astrophysical applications, such as RMHD coronal models, ${ }^{7-13}$ as well as plasma fusion studies (where RMHD was introduced originally ${ }^{1,3}$ ).

It is clear then that the subject of applicability and validity of the RMHD approximation is important for a number of plasma physics applications. In a previous paper ${ }^{14}$ we 
have examined the self-consistency of the RMHD approximation through direct numerical simulations of the RMHD equations, which showed that the approximation does not invalidate itself as the system evolves in time. That study shows that the turbulent energy cascade along the direction of the mean magnetic field is self-limited, and therefore the RMHD assumption of weak spatial variations in the parallel direction persists in time. Another study addressing that issue within RMHD was discussed in Ref. 15. The purpose of the present paper is to give new support to the use of the RMHD approximation now by direct comparison of simulations of the RMHD equations and the full compressible MHD equations at low Mach number using several types of initial conditions. Results will be compared one to one for global quantities, such as mean energies, square current, and vorticity as for energy spectra and direct visualization of currents. The separation (mean-squared-normalized distance) between the two solutions will be computed for different values of the mean magnetic field and initial conditions.

Although the cases examined are specific, the results shown here give strong support for the use of RMHD when the conditions for its validity are satisfied, as well as good support for situations in which some of these conditions are partially relaxed. In the latter case we find the interesting property that the realm of applicability of RMHD can be enhanced by the dynamical reinforcement of the lowfrequency requirement, achieved through anisotropic cascade.

The organization of the paper is as follows. Section II describes the equations for both compressible MHD and reduced MHD, along with the codes to numerically solve them. Section III presents the numerical results for different types of initial conditions. Section IV contains the conclusions.

\section{MODEL AND EQUATIONS}

\section{A. Compressible MHD}

The macroscopic description of a plasma given by compressible three-dimensional (3D) MHD (CMHD3D) involves a fluctuating flow velocity $\mathbf{v}(x, y, z, t)$, magnetic field $\mathbf{B}(x, y, z, t)$, and density $\rho(x, y, z, t)$. The MHD equations are

$$
\begin{aligned}
& \frac{\partial \mathbf{v}}{\partial t}+(\mathbf{v} \cdot \nabla) \mathbf{v}=-\frac{1}{\rho} \nabla p+\frac{(\nabla \times \mathbf{B}) \times \mathbf{B}}{4 \pi \rho} \\
&+\nu\left(\nabla^{2} \mathbf{v}+\frac{1}{3} \nabla \nabla \cdot \mathbf{v}\right), \\
& \frac{\partial \mathbf{B}}{\partial t}=\nabla \times(\mathbf{v} \times \mathbf{B})+\mu \nabla^{2} \mathbf{B},
\end{aligned}
$$

where $p$ is the pressure, $\nu$ is the kinematic viscosity, and $\mu$ is the magnetic diffusivity. A continuity equation for the density $\rho$ and an equation of state complete the system. We treat the pressure as polytropic $p / p_{0} \sim\left(\rho / \rho_{0}\right)^{\gamma}, \gamma=5 / 3$, and consider a regime with low Mach number $M_{s}=v_{0} / c_{s}=0.25$, where $v_{0}$ is the initial rms velocity fluctuation value and $c_{s}$ $=\sqrt{\gamma p_{0} / \rho_{0}}$ is the sound speed, with $p_{0}$ and $\rho_{0}$ the initial pressure and density, respectively.
A mean background magnetic field (dc field) along the $z$ direction, $\mathbf{B}_{0}=B_{0} \hat{\mathbf{z}}$, is assumed so that the total magnetic field is $\mathbf{B}=B_{0} \hat{\mathbf{z}}+\mathbf{b}$ where the fluctuating magnetic field is $\mathbf{b}$. Then the MHD Eqs. (1) and (2) can be written in the form

$$
\begin{aligned}
& \frac{\partial \mathbf{v}}{\partial t}+(\mathbf{v} \cdot \nabla) \mathbf{v}=-\frac{1}{\rho} \nabla\left(p+\frac{B^{2}}{8 \pi}\right)+\frac{B_{0} \nabla_{z} \mathbf{b}}{4 \pi \rho}+\frac{(\mathbf{b} \cdot \nabla) \mathbf{b}}{4 \pi \rho} \\
&+\nu\left(\nabla^{2} \mathbf{v}+\frac{1}{3} \nabla \nabla \cdot \mathbf{v}\right), \\
& \frac{\partial \mathbf{B}}{\partial t}=B_{0} \nabla_{z} \mathbf{v}+(\mathbf{b} \cdot \nabla) \mathbf{v}-\mathbf{v} \cdot \nabla \mathbf{b}-\mathbf{B}(\nabla \cdot \mathbf{v})+\mu \nabla^{2} \mathbf{B},
\end{aligned}
$$

where the mean magnetic field $B_{0}$ appears explicitly and $\nabla_{z}$ is the gradient along its direction. This form is useful for comparison with the RMHD equations.

\section{B. Reduced MHD}

The RMHD limit attempts to describe compressible MHD flows in which (1) the flow becomes incompressible or nearly so, (2) the mean background magnetic field $B_{0}$ is large when compared to magnetic- and velocity-field fluctuations values, ${ }^{16}$ and (3) the dynamical processes of interest occur at slow convective time scales, well separated from highfrequency waves.

The latter condition, recognized explicitly in Montgomery's derivation ${ }^{2}$ from the incompressible equations, may be quantified by defining the Fourier modes that are expected to follow the RMHD equations according to the inequality

$$
\tau_{\mathrm{nl}}(\mathbf{k})<\tau_{A}(\mathbf{k}) \text {. }
$$

Here $\tau_{\mathrm{nl}}(\mathbf{k})=1 /\left(k v_{k}\right)$ is the nonlinear time associated with the speed $v_{k}$ at wave number $k$ and $\tau_{A}(\mathbf{k})=1 /\left(k_{\|} V_{A_{0}}\right)$ is the Alfvén time, where $V_{A_{0}}=B_{0} / \sqrt{4 \pi \rho_{0}}$ is the Alfvén speed. Condition (5) is related to the critical balance criterion, ${ }^{17}$ in which the approximate equality of the two time scales holds for the steady inertial range modes in anisotropic incompressible MHD. Although the approximate equality might hold for a range of modes in a plasma, one should not expect that this is true for all the modes in the system or all conditions (see Ref. 14 for a more complete discussion of this issue). An interesting example is the solar corona, where through driving at the coronal base the inequality can be maintained at the low- $k$ modes in the system. ${ }^{12}$

The strong magnetic field $B_{0} \rightarrow \infty$ limit is formally singular, as the frequencies of the turbulence, at convective time scales, become increasingly separated from the Alfvenic wave frequencies. To maintain the RMHD time scale inequality in this limit, the condition that nonlinear terms [e.g., $(\mathbf{b} \cdot \nabla) \mathbf{b}]$ are comparable in size to or smaller than the linear ones (e.g., $B_{0} \nabla_{z} \mathbf{b}$ ) implies that it is necessary to restrict gradients along $B_{0}$ to be small when compared to gradients in the perpendicular directions.

Further technical complications arise when RMHD is derived formally, starting from a compressible MHD model, and these have practical ramifications as we see below in later sections. Magnetoacoustic couplings are present, and now the relevant dimensionless ratio is the Mach number 
associated with the turbulent fluctuations, $M_{s}$, which $\rightarrow 0$ in the incompressible limit. This is also a singular limit, in which the convective motions and wave activity have divergent characteristic time scales. Consequently there are special conditions that must be formally imposed on the system when the mathematical limits $b / B_{0} \rightarrow 0$ (rms fluctuating field $b)$ and $M_{s} \rightarrow 0$ are imposed simultaneously, if the dynamics is to be dominated by low-frequency convective motion. ${ }^{4} \mathrm{As}$ in the limit of incompressible hydrodynamics, ${ }^{18,19}$ a proper approach to such a singular limit requires that conditions be placed on initial data to restrict the level of "fast" wave activity, thus producing solutions that remain within the realm of the "reduced" dynamical equations for finite time. In particular, the frequency of Alfvénic couplings at a given wave number is reduced by imposing the spectral anisotropy, so that $\omega=k_{\|} V_{A} \ll k V_{A}$. However, for magnetosonic couplings, which involve nonzero parallel variance, $\omega \sim k V_{A}$ for low plasma beta (strong $B_{0}$ ) and therefore variance anisotropy, i.e., parallel component fluctuations smaller than perpendicular component fluctuations, is required to maintain the convective time scale restriction needed for RMHD (see Ref. 4).

For the description by the RMHD model this implies a combination of factors-limits on magnetoacoustic activity, low Mach number, and strong mean magnetic field. Here we will be concerned not with an examination of the formal asymptotic properties but rather with the practical issue of how well the RMHD model performs in real simulations.

Providing the necessary restrictions are met, the above conditions result in a set of leading-order equations ${ }^{1,2,4}$ involving incompressible plane-polarized velocity and magnetic-field fluctuations $\mathbf{v}(x, y, z, t)=\left(v_{x}, v_{y}, 0\right)=\mathbf{v}_{\perp}$, $\mathbf{b}(x, y, z, t)=\left(b_{x}, b_{y}, 0\right)=\mathbf{b}_{\perp}$,

$$
\begin{aligned}
& \frac{\partial \mathbf{v}_{\perp}}{\partial t}+\left(\mathbf{v}_{\perp} \cdot \nabla_{\perp}\right) \mathbf{v}_{\perp}=-\frac{1}{\rho} \nabla_{\perp}\left(p+\frac{B^{2}}{8 \pi}\right)+\frac{B_{0} \nabla_{z} \mathbf{b}_{\perp}}{4 \pi \rho} \\
&+\frac{\left(\mathbf{b}_{\perp} \cdot \nabla_{\perp}\right) \mathbf{b}_{\perp}}{4 \pi \rho}+\nu \nabla_{\perp}^{2} \mathbf{v}_{\perp}, \\
& \frac{\partial \mathbf{b}_{\perp}}{\partial t}=B_{0} \nabla_{z} \mathbf{v}_{\perp}+\left(\mathbf{b}_{\perp} \cdot \nabla_{\perp}\right) \mathbf{v}_{\perp}-\left(\mathbf{v}_{\perp} \cdot \nabla_{\perp}\right) \mathbf{b}_{\perp}+\mu \nabla_{\perp}^{2} \mathbf{b}_{\perp}
\end{aligned}
$$

with $\nabla_{\perp} \cdot \mathbf{v}_{\perp}=0$, where $\nabla_{\perp}=\left(\partial_{x}, \partial_{y}, 0\right)$ is the gradient in the transverse directions and $\rho=\rho_{0}$.

In this form, it can be seen that some of the nonlinear terms in CMHD3D are not present in RMHD (e.g., those involving parallel component fluctuations) and also that fluctuations are incompressible in this approximation. However, the RMHD equations remain strongly nonlinear and should not be confused with any kind of linear small amplitude fluctuation limit.

The fact that the fluctuations are incompressible in RMHD implies that the pressure can be eliminated from the equations, which can be put in a form involving two scalar fields, ${ }^{1}$ the fluid vorticity $\omega_{z}(x, y, z, t)$, and the magnetic vector potential $a_{z}(x, y, z, t)$ as

$$
\frac{\partial \omega_{z}}{\partial t}+(\mathbf{v} \cdot \nabla) \omega_{z}=\frac{B_{0} \nabla_{z} j_{z}}{4 \pi \rho}+\frac{\left(\mathbf{b} \cdot \nabla_{\perp}\right) j_{z}}{4 \pi \rho}+\nu \nabla_{\perp}^{2} \omega_{z},
$$

$$
\frac{\partial a_{z}}{\partial t}+(\mathbf{v} \cdot \nabla) a_{z}=B_{0} \nabla_{z} \psi_{z}+\mu \nabla_{\perp}^{2} a_{z}
$$

where $\omega_{z}=-\nabla_{\perp}^{2} \psi_{z}$ and $j_{z}=-\nabla_{\perp}^{2} a_{z}$, with $\psi_{z}$ the stream function and $j_{z}$ the current density. The velocity and magnetic fluctuation fields are obtained from the scalar potentials as $\mathbf{v}_{\perp}=\nabla \times \psi_{z} \hat{\mathbf{z}}$ and $\mathbf{b}_{\perp}=\nabla \times a_{z} \hat{\mathbf{z}}$, respectively.

The leading-order current density in RMHD is $\mathbf{j}$ $=\left(0,0, j_{z}\right)$ that is directly aligned with the mean magnetic field. Higher-order contributions to the current (coming from weak $\nabla_{z}$ terms) are corrections to RMHD and are not considered here. Similarly, the leading-order (parallel) component of the vorticity is indicated by $\omega_{z}$.

Velocity and magnetic fluctuation components along the mean magnetic-field direction are not present in the formulated equations. It is possible however to retain those fluctuations and the equations for them have a passive form. ${ }^{2,4}$ By that, we mean that they do not appear in the equations for the transverse components which evolve independently and the equations for these parallel components are linear once the transverse fluctuations are known.

\section{Numerical codes}

We employ different pseudospectral codes to solve each set of equations. Triply periodic boundary conditions are assumed in a cube of side $2 \pi L$ (where $L \sim 1$ is the initial correlation length of the fluctuations, defined as the unit length). In the codes, the Fourier components of the fluctuations are evolved in time, starting from a specified set of the Fourier modes (see the following section for the different types of initial conditions), with given total energy and random phases. The RMHD code specifies exactly the same initial fluctuation fields (velocity and magnetic field) as prepared in the full MHD code, except in cases where parallel component fluctuations are included in CMHD3D but are not present in RMHD.

The same resolution is used in both codes in all three directions in most of the runs presented here, which have a moderate resolution of $64^{3}$ Fourier modes, allowing many different runs to be done with different initial conditions and/or mean magnetic field. The kinetic and magnetic Reynolds numbers are defined as $R=1 / \nu$ and $R_{m}=1 / \mu$, respectively, based on unit initial rms velocity fluctuation, unit length, and nondimensional values for the viscosity and diffusivity. Here we considered $R=R_{m}=200$ in most of the runs. A particular CMHD3D run is made also at $128^{3}$ and Reynolds numbers of $R=R_{m}=400$ and compared to a RMHD run with $128 \times 128 \times 16$ resolution (i.e., fewer modes along the mean magnetic-field direction).

A second-order Runge-Kutta time integration is performed, the nonlinear terms evaluated using the standard pseudospectral procedure. ${ }^{20}$ The runs are freely evolved for 10 or 20 unit times (the initial eddy turnover time defined in terms of the initial unit rms velocity fluctuation and unit length). Different values of the magnetic field $B_{0}=1,2,4$, and 8 are considered (in units of the initial rms magnetic fluctuation value). 


\section{RESULTS AND DISCUSSION}

\section{A. Anisotropic initial conditions}

The first set of initial conditions we considered in this study is generated using a two-step process. First, a set of Fourier modes for both magnetic- and velocity-field fluctuations is produced, with amplitudes such that the (omnidirectional) energy spectrum is a Kolmogorov spectrum proportional to $k^{-5 / 3}$ for $1 \leqslant k \leqslant 16$ and Gaussian random phases. Modes outside of this range in $k$ space are set to 0 . Second, an anisotropic filter is applied, so that excited modes for which $\tau_{\mathrm{nl}}(\mathbf{k})>\tau_{A}(\mathbf{k})$ are initially set to 0 . This is to ensure that only modes which satisfy the RMHD requirement Eq.

(5) are excited initially.

Taking the Kolmogorov inertial range form $v_{k} \sim k^{-1 / 3}$ into account, this condition becomes

$$
k_{\|} \leqslant C \frac{k^{2 / 3}}{V_{A_{0}}}
$$

where $C$ is a $O(1)$ constant. As discussed above, this form is similar to the critical balance condition for anisotropic MHD discussed in Ref. 17 (whereas, instead, the approximate equality holds). Here we view this inequality as one of the conditions for the validity of the RMHD approximation. For marginal attainment of the time scale inequality, we consider runs with $C=1$ (see, however, some additional cases below). The condition means that only large, parallel wavelength modes (low $k_{\|}$) are allowed in the initial fluctuations and becomes increasingly restrictive as the value of the mean magnetic field (i.e., $V_{A_{0}}$ ) is increased.

To complete the specification of the initial conditions, the fluctuations are normalized so that the initial meansquare values of the magnetic and velocity fields are both equal to 1 (unit value). Cross and magnetic helicities are initialized at very small values (remind that if the normalized cross helicity is unity, there is no turbulent dynamics). Only plane-polarized fluctuations are considered in this case, that is, fluctuations are perpendicular to the mean magnetic field, e.g., $\mathbf{v}(\mathbf{x}, t=0)=\mathbf{v}_{\perp}(\mathbf{x}, 0)$.

The runs performed throughout this paper do not contain any magnetic or velocity stirring terms, so that the CMHD3D and RMHD systems freely evolve during several nonlinear times until $t=10$. We performed runs for $B_{0}=1,2$, and 4. Global quantities for the cases $B_{0}=4$ and $B_{0}=1$, such as mean-square magnetic-field fluctuations, velocity field, total current, and total vorticity, are shown as functions of time in Figs. 1 and 2. The CMHD3D and RMHD solutions are almost indistinguishable from one another for the $B_{0}=4$ case. The solutions can be distinguished for the $B_{0}=1$ case, although the RMHD case still tracks reasonably well the behavior of the global quantities shown. We want to remark that the magnetic-field and velocity-field quantities for the CMHD3D case include also the parallel fluctuations components that may have developed in the system evolution. In the case of the current and vorticity, transverse components are also considered for the CMHD3D case (not present in the leading-order RMHD system).

A more quantitative and detailed way of measuring the (a)

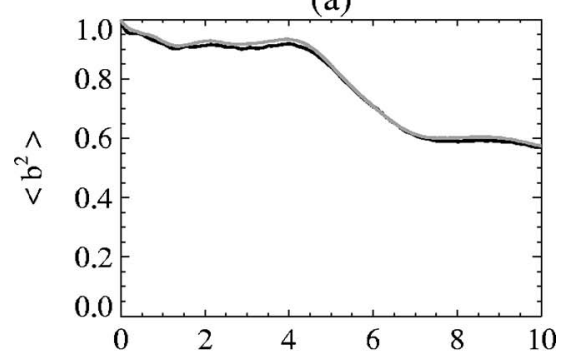

(b)

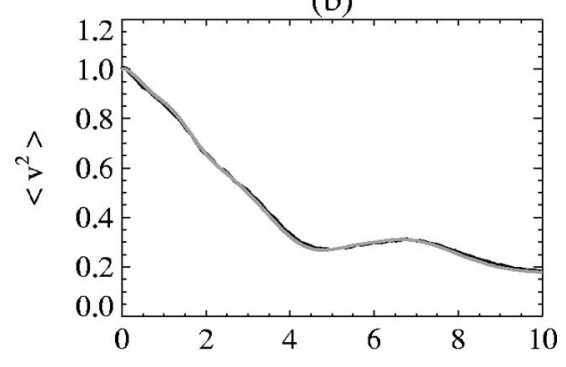

(c)

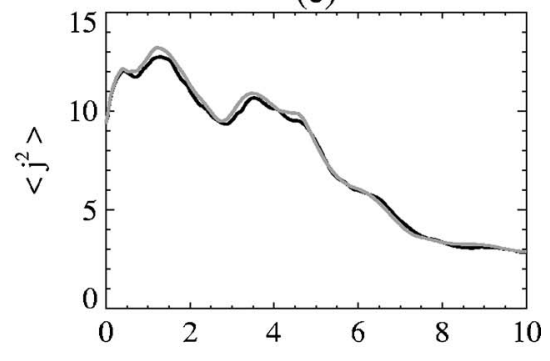

(d)

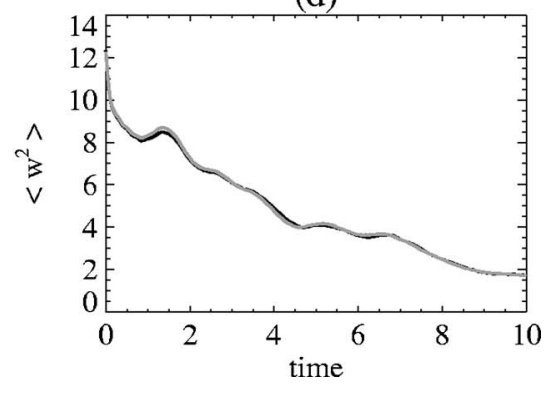

FIG. 1. (a) Mean-square fluctuating magnetic field, (b) velocity field, (c) current density, and (d) vorticity as functions of time for the case $B_{0}=4$, anisotropic initial conditions $\left(k_{\|}<k_{\perp}^{2 / 3} / V_{A_{0}}\right)$, and plane-polarized fluctuations. CMHD3D solution (dark line) and RMHD solution (light line).

distance between the two solutions is to compute the pointto-point separation between the two solutions (the squared "distance" between them), defined as

$\mathcal{E}=d^{2}=\frac{\int\left(\mathbf{b}^{\mathrm{RMHD}}-\mathbf{b}^{\mathrm{CMHD} 3 \mathrm{D}}\right)^{2}+\left(\mathbf{v}^{\mathrm{RMHD}}-\mathbf{v}^{\mathrm{CMHD} 3 \mathrm{D}}\right)^{2} d^{3} x}{\int\left(\mathbf{b}^{\mathrm{CMHD} 3 \mathrm{D}}\right)^{2}+\left(\mathbf{v}^{\mathrm{CMHD} 3 \mathrm{D}}\right)^{2} d^{3} x}$,

where the integral can be numerically computed as a sum of the Fourier modes. It should be noted that this quantity is a 
(a)

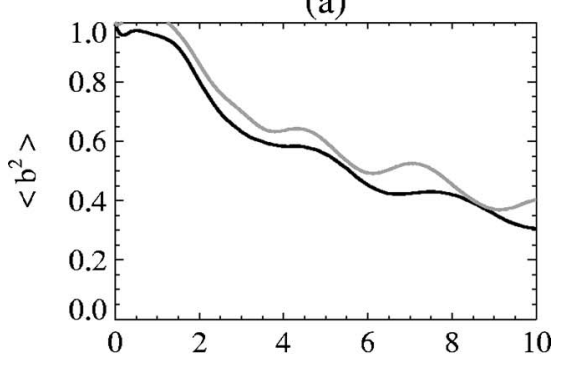

(b)

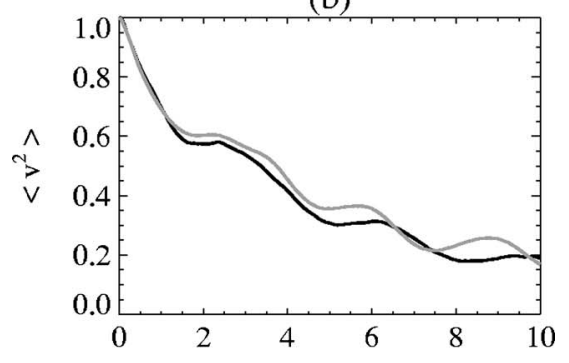

(c)

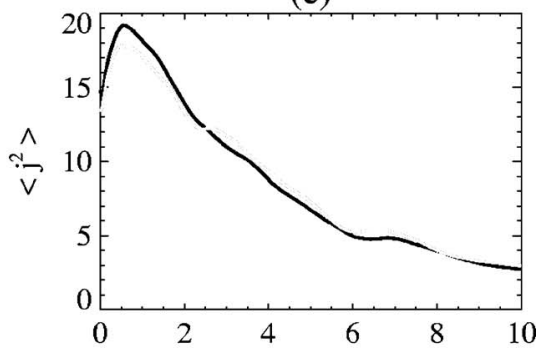

(d)

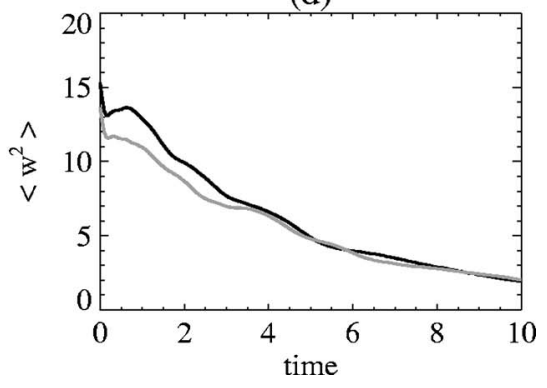

FIG. 2. (a) Mean-square fluctuating magnetic field, (b) velocity field, (c) current density, and (d) vorticity as functions of time for the case $B_{0}=1$, anisotropic initial conditions $\left(k_{\|}<k_{\perp}^{2 / 3} / V_{A_{0}}\right)$, and plane-polarized fluctuations. CMHD3D solution (dark line) and RMHD solution (light line).

very sensitive measurement of the proximity of the two solutions since simple phase differences between the solutions would be seen as large values for $\mathcal{E}$. In this regard, the global quantities comparison (and energy spectra shown below) is a more adequate measurement of the statistical proximity of the two solutions even in cases where the separation, as defined above, is large. We want, however, to compute this quantity as a high demanding measurement of the proximity of the two solutions.

The separation is shown as a function of time and for

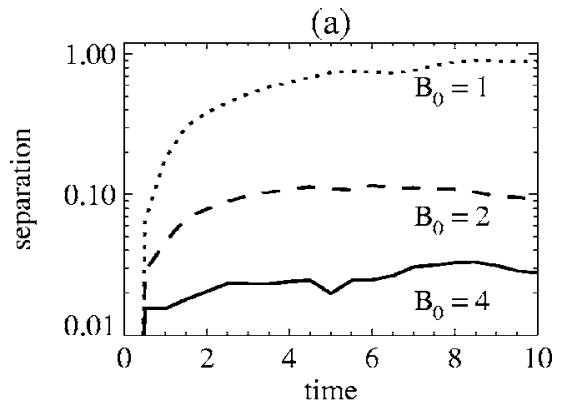

(b)

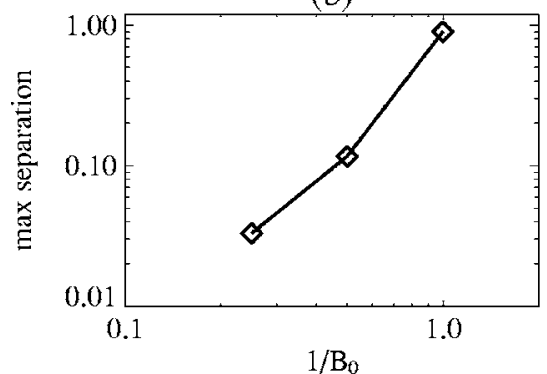

FIG. 3. (a) Separation between the RMHD solution and the CMHD3D solution as a function of time (see text) with anisotropic initial conditions (as described in Fig. 1), for different values of the mean field: $B_{0}=4$ (continuous line), $B_{0}=2$ (dashed line), and $B_{0}=1$ (dotted line); (b) Maximum separation between the RMHD solution and the CMHD3D solution as a function of $1 / B_{0}$.

different values of $B_{0}$ in Fig. 3. The maximum separation is also plotted for three different values of $B_{0}$ as a function of $1 / B_{0}$.

The separation remains less than $3 \%$ for the case $B_{0}=4$, $10 \%$ for the case $B_{0}=2$, and increases to $90 \%$ for $B_{0}=1$. As can be concluded from Figs. 1-3 the results show that if the appropriate anisotropic set of initial conditions are chosen, the solution given by RMHD remains very close to the one given by CMHD3D for the cases where $B_{0}$ is larger than the fluctuations level (i.e., $B_{0}>1$ ).

The agreement between the solutions for the case $B_{0}$ $=1$ can actually be made to improve considerably if a more restrictive anisotropic initial condition is applied, and instead of $C=1$ we use values $C=1 / 2$ and $C=1 / 4$ for the constant in the RMHD condition. The separations between the CMHD3D and RMHD solutions for these three cases are shown in Fig. 4(a). Figure 4(b) shows the maximum distance as a function of $C$ (whose inverse value measures the degree of the initial anisotropy). These results show that if the mean magnetic field is fixed (in this case $B_{0}=1$ ), the agreement between RMHD and CMHD3D is improved as more anisotropic initial conditions are considered. This is due to the further reduction of high-frequency modes in lower- $k_{\|}$initial data.

\section{B. Isotropic, large-scale initial conditions}

In this case we consider the initial Fourier modes in a shell in $k$ space $1 \leqslant k \leqslant 2$ at low wave numbers, with constant amplitudes and random phases. Unlike the previous case, no initial anisotropic filter is applied here, so the initial conditions are spectrally isotropic. In terms of the linear modes of 
(a)

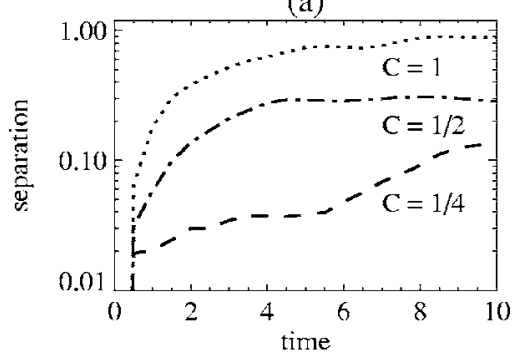

(b)

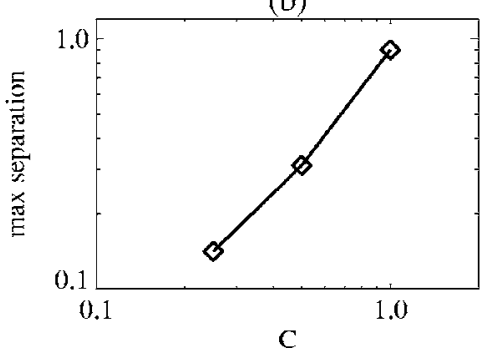

FIG. 4. (a) Separation between the RMHD solution and the CMHD3D solution as a function of time for the case $B_{0}=1$ and different values of the constant $C$ in the anisotropic condition $k_{\|}<C k_{\perp}^{2 / 3} / V_{A_{0}}: C=1$ (dotted line), $C=1 / 2$ (dash-dotted line), and $C=1 / 4$ (dashed line). (b) Maximum separation between the RMHD solution and the CMHD3D for the previous case as a function of the constant $C$ in the anisotropic condition.

the system, the Fourier modes with wave-number magnitude $k$ include contributions with frequencies varying from 0 to $k V_{A}$. As above, only plane-polarized fluctuations (transverse to the mean magnetic field) are included, so these are (lowto-high-frequency) Alfvén mode fluctuations and not magnetosonic modes. We maintain other parameters and initial conditions, such as mean-square values of magnetic and velocity fluctuations set to 1 , low cross helicity, and no magnetic helicity.

This set of initial conditions represents a more general situation that may arise in an application, where no initial spectral anisotropy is imposed (for instance, they could represent better the effect of isotropic driving). Since only low$k$ modes are excited initially, we expect an energy cascade to develop and populate the large wave-number modes in a few turbulent times. Strong nonlinear activity is expected.

We perform simulations with $B_{0}=1,2,4$, and 8 . The evolution of global quantities is shown in Fig. 5 for the case $B_{0}=8$. The CMHD3D and RMHD solutions keep remarkably close throughout. The fast oscillations observed in the meansquare magnetic- and velocity-field values are consequences of the Alfvén waves $\left(\omega=k_{\|} V_{A_{0}}\right)$ excited during the evolution of the system. The RMHD solutions track this time behavior well and similarly for the fluctuations observed in the meansquare current and vorticity. The evolution of global quantities for the case $B_{0}=1$ is shown in Fig. 6. Here the agreement has worsened as compared to the $B_{0}=8$ case, but the general behavior is still well tracked.

The separation between the two solutions as function of time is shown in Fig. 7(a) for different values of $B_{0}$. Figure $7(\mathrm{~b})$ shows the maximum separation as a function of $1 / B_{0}$. Although errors are larger than in the anisotropic cases shown in Sec. III A, the solutions are quite close to each (a)

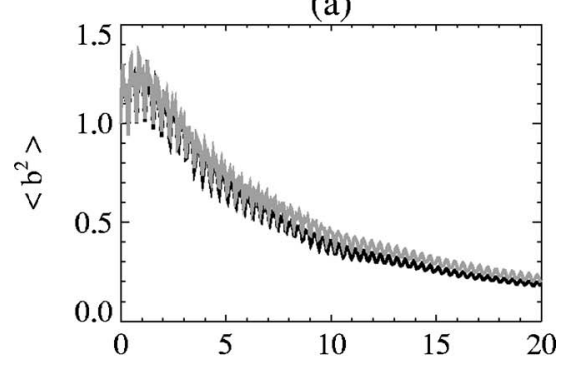

(b)

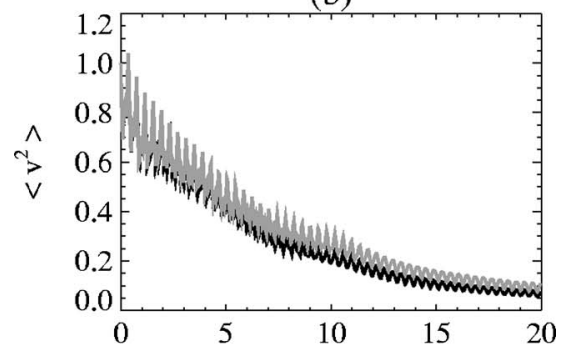

(c)

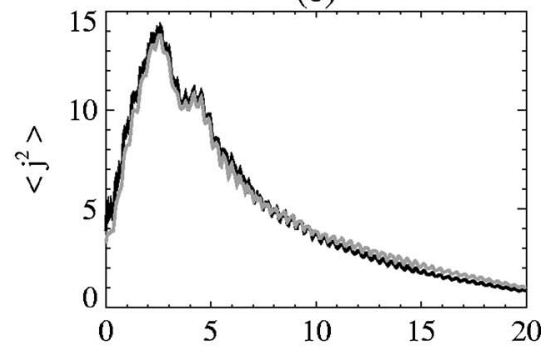

(d)

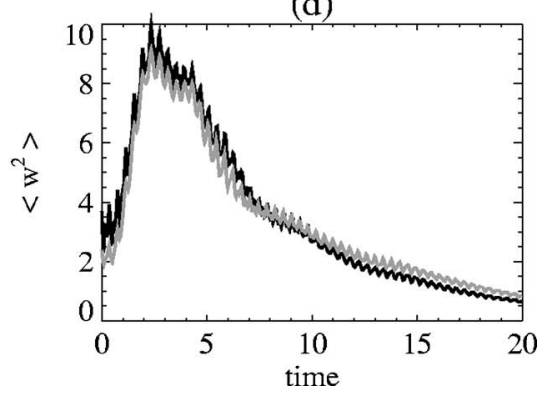

FIG. 5. (a) Mean-square fluctuating magnetic field, (b) velocity field, (c) current density, and (d) vorticity as functions of time for the case $B_{0}=8$, isotropic initial conditions, and plane-polarized fluctuations. CMHD3D solution (dark line) and RMHD solution (light line).

other for the large $B_{0}$ cases. For the largest $B_{0}=8$ case the separation between the solutions remains smaller than $20 \%$ at late times (when the energy of the system is low) and is smaller than $5 \%$ at short times, when the peak of dissipation occurs (see Fig. 5). The plasma $\beta$ for the $B_{0}=8$ case is $\beta$ $=c_{s}^{2} / V_{A_{0}}^{2}=0.25$ which is the lowest value we achieved in these runs (other $B_{0}=1,2$, and 4 cases have $\beta=16,4$, and 1 , respectively).

In this series of runs, the initial state includes modes that do not satisfy the RMHD condition, but the RMHD equations fare somewhat better in comparison to CMHD3D than 
(a)

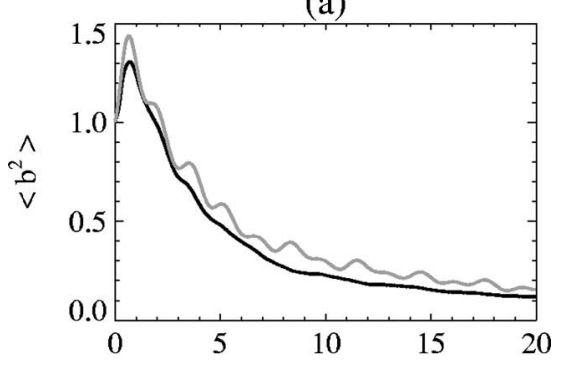

(b)

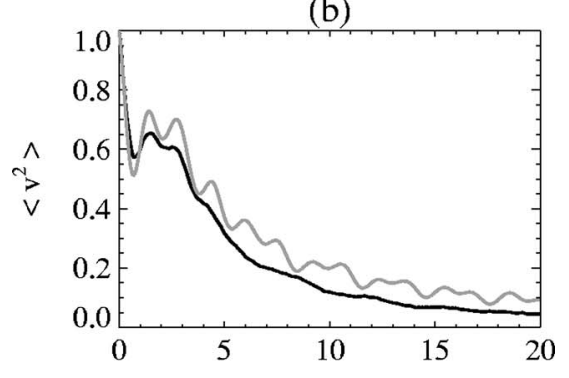

(c)

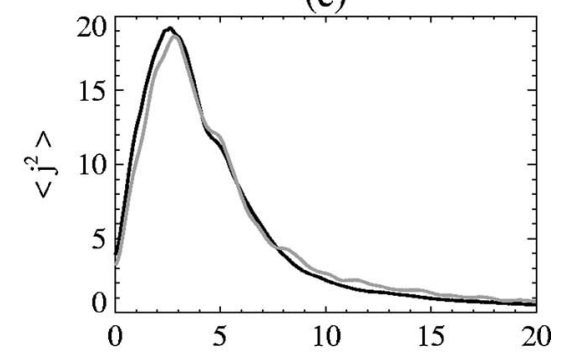

(d)

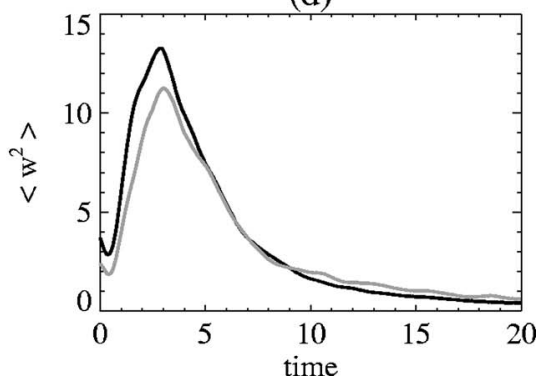

FIG. 6. (a) Mean-square fluctuating magnetic field, (b) velocity field, (c) current density, and (d) vorticity as functions of time for the case $B_{0}=1$, isotropic initial conditions, and plane-polarized fluctuations. CMHD3D solution (dark line) and RMHD solution (light line).

might have been expected. One reason for this is related to the dynamical enhancement of spectral anisotropy, caused by a strong mean magnetic field. ${ }^{21}$ The energy cascade is stronger in the transverse directions than in the parallel one, and so there is less energy in large parallel wave-number modes than in large perpendicular wave-number modes. Development of spectral anisotropy in the presence of a strong mean magnetic field has been observed in numerical simulations of both incompressible and compressible $\mathrm{MHD}^{22,23}$ (and recently also in supersonic compressible MHD as shown in Ref. 24). Here we note that the cascade is predominantly in (a)

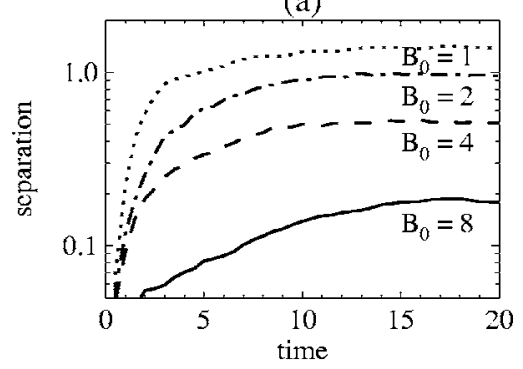

(b)

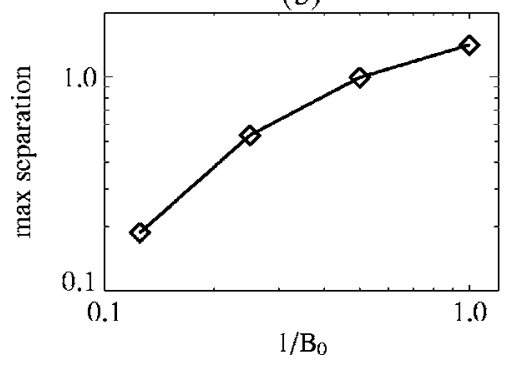

FIG. 7. (a) Separation between the RMHD solution and the CMHD3D solution as a function of time with isotropic initial conditions, for different values of the mean field: $B_{0}=8$ (continuous line), $B_{0}=4$ (dashed line), $B_{0}$ $=2$ (dash-dotted line), and $B_{0}=1$ (dotted line); (b) Maximum separation between the RMHD solution and the CMHD3D solution as a function of $1 / B_{0}$.

the direction of higher $k_{\perp}$ and at nearly constant $k_{\|}$, and this means that as time passes, excitation that is initially outside the RMHD regime may enter into the RMHD region defined by Eq. (5). In this way anisotropic spectral transfer helps, in part, to maintain the conditions needed for validity of RMHD and thus helps to make it possible for the two solutions to remain close to one another.

We investigate the anisotropy development for the $B_{0}$ $=8$ case in more detail and perform a larger resolution $128^{3}$ CMHD3D run, halving the viscosity and resistivity (so the effective kinetic and magnetic Reynolds numbers are 400). This is both for verification and to obtain more extended energy spectra (see below). Furthermore, the RMHD run for this comparison case has been performed with $128 \times 128$ $\times 16$ resolution, that is, a smaller number of modes in the parallel direction. Time histories of several global quantities are shown in Fig. 8. The RMHD solution tracks the CMHD3D solution quite well, even in some detailed time behavior. Again, the observed oscillations in the magnetic and velocity mean-square values are due to the presence of the parallel propagating Alfvén waves, captured by both CMHD3D and RMHD.

Energy spectra for this comparison case are shown in Fig. 9 at a time $t=4$, corresponding to the peak of meansquare current and vorticity (spectra at later times are similar to the one shown here). Figure 9(a) shows the omnidirectional total- (kinetic plus magnetic) energy spectrum $E(k)$ for both CMHD3D and RMHD. The spectrum is broadband and has developed from the initial narrow $1 \leqslant k \leqslant 2$ initial state, as a result of the turbulent cascade. Only at very large $k$ can the CMHD3D spectrum be distinguished from the RMHD one. Figure 9(b) shows the perpendicular spectrum $E\left(k_{\perp}\right)$. 
(a)

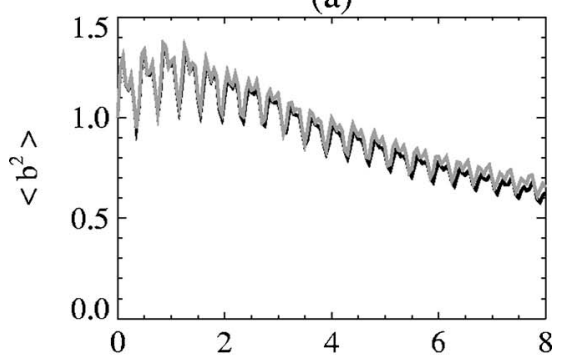

(b)

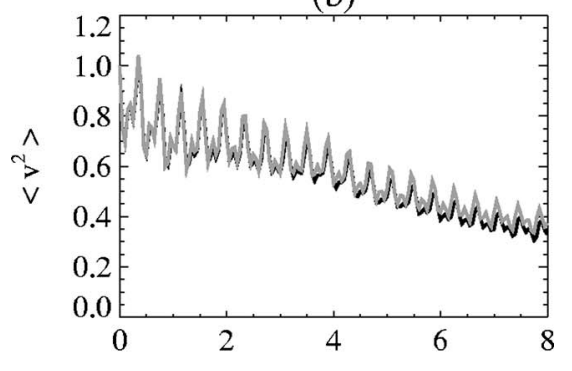

(c)

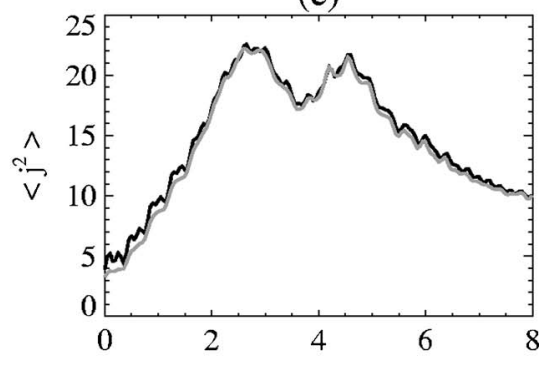

(d)

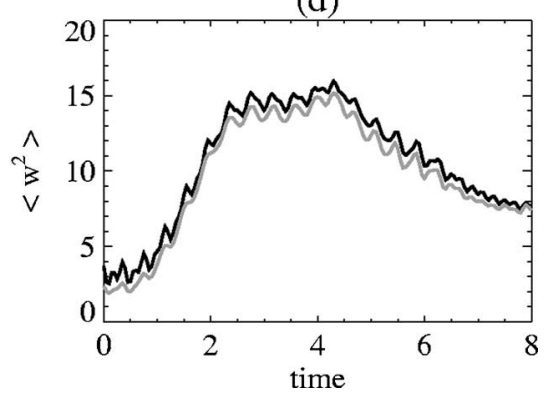

FIG. 8. (a) Mean-square fluctuating magnetic field, (b) velocity field, (c) current density, and (d) vorticity as a function of time for the case $B_{0}=8$, isotropic initial conditions and plane polarized fluctuations. CMHD3D solution at $128^{3}$ resolution (dark line) and RMHD solution at $128 \times 128 \times 16$ resolution (light line).

This spectrum seems to be consistent with a Kolmogorov $k_{\perp}^{-5 / 3}$ law although in a very limited range in $k_{\perp}$ due to the lack of numerical resolution. In Fig. 9(c) we show the parallel energy spectrum $E\left(k_{\|}\right)$(in linear-log scale). As expected from the anisotropy development, the parallel energy spectrum remains much smaller than the perpendicular spectrum, especially at large wave numbers. The spectrum obtained from RMHD tracks the spectrum obtained from CMHD3D very well at low wave-numbers. The parallel spectrum in the

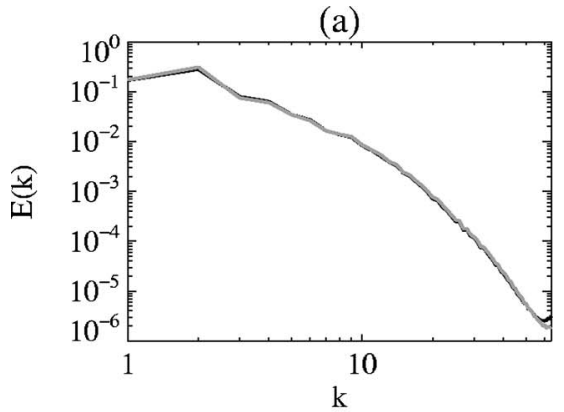

(b)

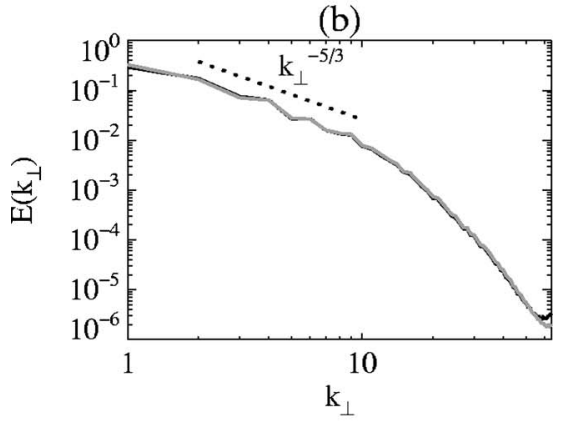

(c)

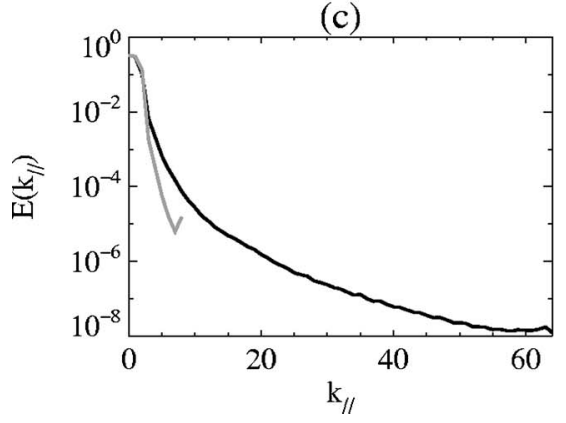

FIG. 9. (a) Omnidirectional energy spectrum (kinetic plus magnetic) $E(k)$ for the case $B_{0}=8$, isotropic initial conditions, and at $t=4$. CMHD3D solution at $128^{3}$ resolution (dark line), RMHD solution at $128 \times 128 \times 16$ resolution (light line). (b) Perpendicular energy spectrum $E\left(k_{\perp}\right)$; a power law with a slope of $5 / 3$ is indicated with a dotted line. (c) Parallel energy spectrum $E\left(k_{\|}\right)$(in linear-log scale).

RMHD case is truncated due to the smaller number of modes we used in the parallel direction for this particular run.

A visual comparison of structures is made in Fig. 10. This is a cube of three cross sections of the simulation box, showing the spatial structure of the parallel current $j_{z}(x, y, z)$ (along the mean magnetic-field direction) at a particular instant $t=4$. The light tones correspond to upward currents, while the dark tones correspond to downward currents. The same tone scheme is used in both plots from the CMHD3D and the RMHD runs. The anisotropy of the current distribution is evident in this figure, which shows elongated current structures along the mean magnetic-field directions, while variations are strong in the transverse directions. There are only very subtle differences observed in the two figures. At this particular instant of time, the separation between the two solutions is less than 5\%. For comparison we show in Fig. 11 the results for the $B_{0}=1$ case. The top cube [Fig. 11(a)] shows the CMHD3D case at $128^{3}$ resolution, the middle cube [Fig. 11(b)] shows the RMHD case at $128^{3}$ resolution, and the bottom cube [Fig. 11(c)] shows the RMHD case at 

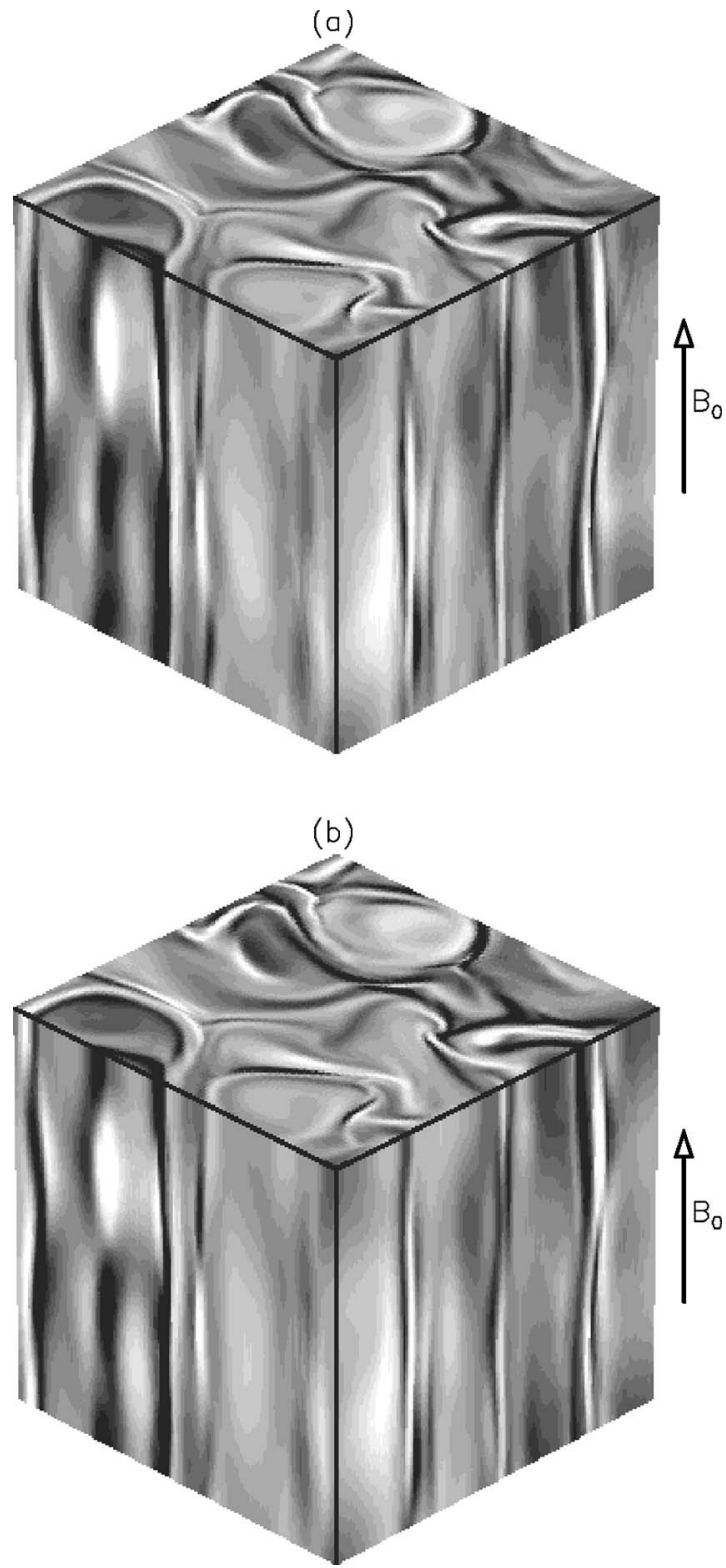

FIG. 10. Cross sections of the parallel current density $j_{z}(x, y, z)$ for the $B_{0}$ $=8$ case, isotropic initial conditions, and at $t=4$; light tones indicate upward currents while dark tones indicate downward currents for the (a) CMHD3D case at $128^{3}$ resolution and (b) RMHD case at $128 \times 128 \times 16$ resolution.

$128 \times 128 \times 16$ resolution. Here the separation between the solutions is about $20 \%$ and more differences can be observed. The RMHD results with fewer modes in the parallel direction appear more different. There is still anisotropy in the current distribution but less developed than in the large $B_{0}=8$ case. Recall that the case shown in Fig. 11 is not the one for which RMHD would be expected to be extremely accurate, and what similarities the current densities show in Figs. 11(a) and 11(c) are somewhat remarkable.

\section{Initial conditions with parallel fluctuations}

Here we consider a set of initial conditions exactly as described in Sec. III B (that is, isotropic, large-scale modes),
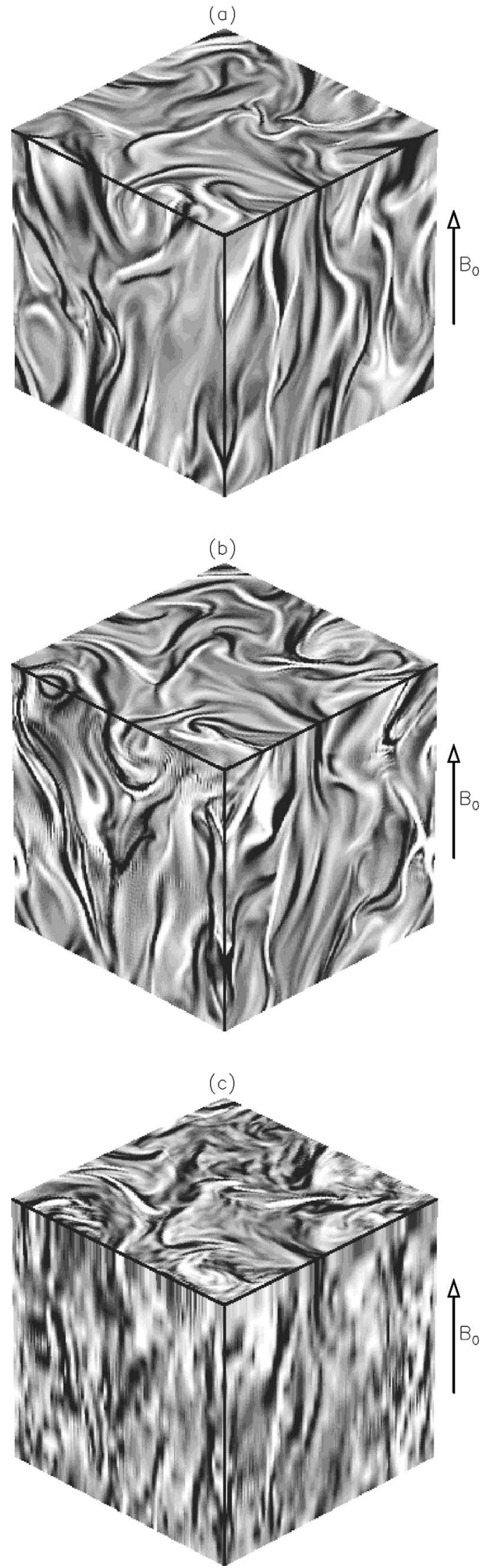

FIG. 11. Cross sections of the parallel current density $j_{z}(x, y, z)$ for the $B_{0}$ $=1$ case, isotropic initial conditions, and at $t=4$; light tones indicate upward currents while dark tones indicate downward currents for the (a) CMHD3D case at $128^{3}$ resolution, (b) RMHD case at $128^{3}$ resolution, and (c) RMHD case at $128 \times 128 \times 16$ resolution. 


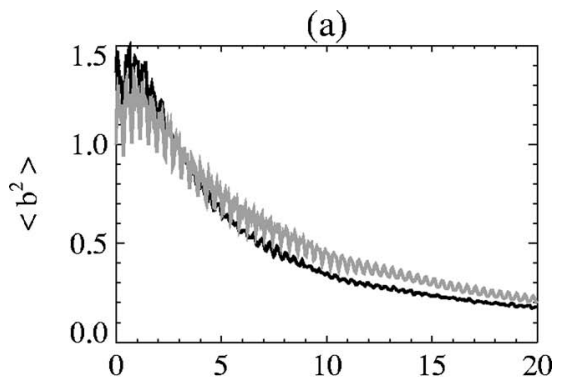

(b)

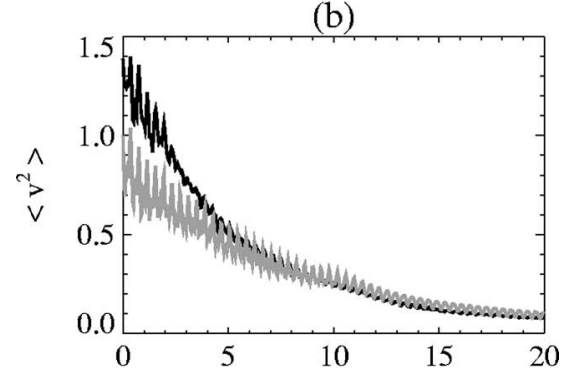

(c)

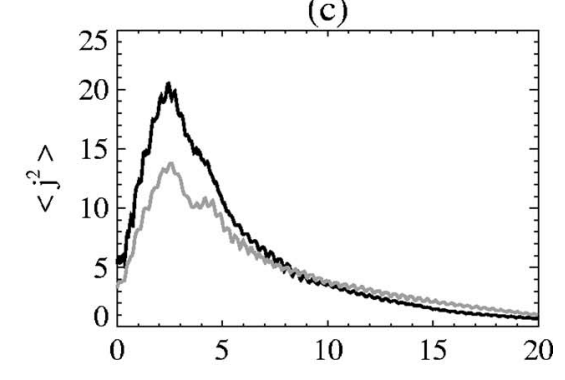

(d)

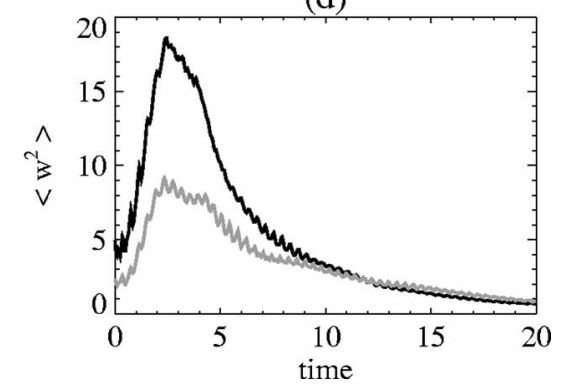

FIG. 12. (a) Mean-square fluctuating magnetic field, (b) velocity field, (c) current density, and (d) vorticity as functions of time for the case $B_{0}=8$, isotropic initial conditions, and plane-polarized fluctuations with $50 \%$ additional initial energy in parallel component fluctuations. CMHD3D solution (dark line) and RMHD solution (light line).

but now parallel fluctuations components to the mean magnetic field are also excited in the CMHD3D case (they are excluded in the RMHD system).

We added $10 \%$ of the total energy in one case and $50 \%$ of the total energy in another case to parallel fluctuation components in the CMHD3D runs. This energy addition is made equipartitioned among magnetic and velocity fluctuations. The global quantities for the case of 50\%-added parallel fluctuations are shown in Fig. 12. The results are compared to the RMHD run which have exclusively

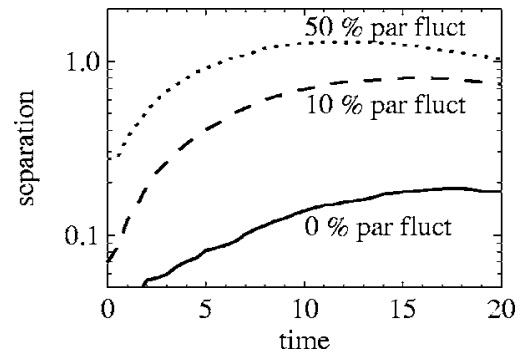

FIG. 13. Distance between the RMHD solution and the CMHD3D solution as a function of time for the case $B_{0}=8$ and different percentages of additional energy in parallel component fluctuations; $0 \%$ (continuous line), $10 \%$ (dashed line), and 50\% (dotted line).

perpendicular fluctuations components (and hence less initial energy). The point-to-point separation between the two solutions is shown in Fig. 13. It is clear that the distance increases as more parallel fluctuations are included initially.

An interesting result is to track the ratio of parallel to perpendicular components for these type of CMHD3D runs. This is shown in Fig. 14. The case without initial parallel fluctuations (corresponding to the case shown in Sec. II B) maintains a low level of parallel fluctuations as the system evolves. The case with initially large parallel fluctuations show, on the other hand, that the ratio increases toward more or less isotropy (recall that the transverse values includes the sum of $x$ and $y$ components). We can conclude from these results that in order for RMHD to keep close to CMHD3D, the amount of initial energy in parallel fluctuations has to be small as compared to the total initial energy. The reason is that parallel fluctuations induce compressive modes (fast and slow magnetosonic modes) in CMHD3D which cannot be tracked with RMHD, where those high-frequency modes are excluded in the approximation. In addition, as we discussed

(a)

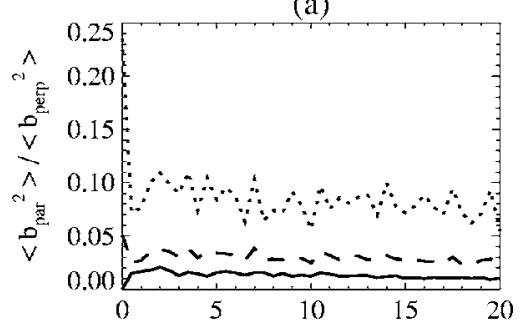

(b)

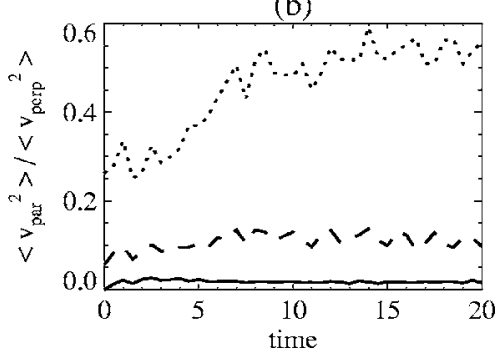

FIG. 14. Energy fraction between parallel and perpendicular component fluctuations for the CMHD3D run with $B_{0}=8$ and different percentages of additional initial energy in parallel component fluctuations: $0 \%$ (continuous line), $10 \%$ (dashed), and 50\% (dotted); (a) magnetic-field fluctuations and (b) velocity-field fluctuations. 
above, only a limited amount of magnetosonic mode activity is consistent with attaining a nearly incompressible solution, ${ }^{4}$ which is an essential feature if RMHD is to be the leadingorder description.

\section{CONCLUSIONS}

We have shown direct numerical comparisons of low Mach number compressible MHD turbulence simulations and reduced MHD simulations. The results can be subdivided into three categories: (a) When initial conditions are constructed to satisfy the RMHD conditions, namely, anisotropic initial conditions and large or moderate mean magnetic fields (which imply that modal nonlinear times are smaller than the modal Alfven times), together with planepolarized component fluctuations, the results show excellent agreement between RMHD and CMHD3D, during evolution over many nonlinear times. (b) If spectrally isotropic initial conditions are instead employed, while maintaining the transverse variance anisotropy associated with the Alfvén mode, the well-known anisotropic MHD cascade induced by a strong mean magnetic field helps to achieve the RMHD conditions as the system evolves in time, and again very good agreement between RMHD and CMHD3D is obtained. (c) When parallel component fluctuations are included in the initial conditions, the agreement between RMHD and CMHD3D worsens, as the amount of energy in parallel component fluctuations is increased, and accordingly the level of magnetoacoustic activity increases.

The main conclusion that can be extracted from these results is that the RMHD approximation remains in very good standing (in the sense that the full MHD solution keeps numerically close to the RMHD solution) when the mathematical conditions assumed for its derivation are satisfied and even in cases in which some of these conditions are not immediately satisfied (spectrally isotropic initial conditions).

Cases where RMHD has been applied include, as mentioned, plasma fusion studies and models of the solar corona. In this last application, for instance, the mean magnetic-field can be very strong, and a large aspect ratio of the geometry is encountered (in a coronal loop or in a coronal hole, variations of the fields along the mean magnetic-field direction are much smaller than variations in the transverse directions). For this situation we can expect that RMHD and CMHD3D solutions will not differ much from each other. This will be especially certain if this type of system is excited by transverse fluctuations (through quasistatic velocity shear driving at the base or by direct injection of shear Alfven waves). It is interesting to note that the results shown in Sec. III C about the addition of parallel fluctuations would suggest that RMHD could still provide a useful base line description in solar wind turbulence, whereas observational results ${ }^{25-28}$ indicate that the ratio of parallel to perpendicular fluctuation energies (power in parallel fluctuations versus total power in perpendicular fluctuations) is approximately 1:10.

Here we have explored a limited range of parameters and initial conditions, but the ones that we believe are the relevant ones for the physical assumptions in RMHD and for the applications encountered in the literature. Although we considered here a basic MHD problem (for instance, it would be interesting to study different types of boundary conditions), the results clearly support the use of RMHD in those applications.

\section{ACKNOWLEDGMENTS}

This research was supported by NASA NAG5-7164, NSF ATM-0105254 and ATM-9977692, and the NZ Marsden Fund 02-UOW-050 MIS.

${ }^{1}$ H. Strauss, Phys. Fluids 19, 134 (1976).

${ }^{2}$ D. C. Montgomery, Phys. Scr., T T2/1, 83 (1982).

${ }^{3}$ B. B. Kadomtsev and O. P. Pogutse, Sov. Phys. JETP 38, 283 (1974).

${ }^{4}$ G. P. Zank and W. H. Matthaeus, J. Plasma Phys. 48, 85 (1992).

${ }^{5}$ A. Bhattacharjee, C. S. Ng, and S. Spangler, Astrophys. J. 494, 409 (1998).

${ }^{6}$ S. E. Kruger, C. C. Hegna, and J. D. Callen, Phys. Plasmas 5, 4169 (1998).

${ }^{7}$ H. Strauss and N. Otani, Astrophys. J. 326, 418 (1988).

${ }^{8}$ D. O. Gómez and C. Ferro Fontán, Astrophys. J. 394, 662 (1992).

${ }^{9}$ D. W. Longcope and R. N. Sudan, Phys. Fluids B 4, 2277 (1992).

${ }^{10}$ G. Einaudi and M. Velli, Phys. Plasmas 6, 4146 (1999).

${ }^{11}$ P. Dmitruk and D. O. Gómez, Astrophys. J. Lett. 527, L63 (1999).

${ }^{12}$ P. Dmitruk and W. H. Matthaeus, Astrophys. J. 597, 1097 (2003).

${ }^{13}$ S. Oughton, P. Dmitruk, and W. H. Matthaeus, Lect. Notes Phys. 614, 28 (2003).

${ }^{14}$ S. Oughton, W. H. Matthaeus, and P. Dmitruk, Phys. Plasmas 11, 2214 (2004).

${ }^{15}$ R. Kinney and J. C. McWilliams, Phys. Rev. E 57, 7111 (1998).

${ }^{16}$ Magnetic and velocity fields are compared by expressing the magnetic field in Alfvén speed units, e.g., $V_{A}^{0}=B_{0} / \sqrt{4 \pi \rho_{0}}$; however, here we retain laboratory units for the magnetic field for clarity.

${ }^{17}$ P. Goldreich and S. Sridhar, Astrophys. J. 438, 763 (1995).

${ }^{18}$ H.-O. Kreis, Commun. Pure Appl. Math. 33, 399 (1980).

${ }^{19}$ S. Klainerman and A. Majda, Commun. Pure Appl. Math. 35, 629 (1982).

${ }^{20} \mathrm{~S}$. Ghosh, M. Hossain, and W. H. Matthaeus, Comput. Phys. Commun. 74, 18 (1993).

${ }^{21}$ J. V. Shebalin, W. H. Matthaeus, and D. Montgomery, J. Plasma Phys. 29, 525 (1983).

${ }^{22}$ S. Oughton, E. R. Priest, and W. H. Matthaeus, J. Fluid Mech. 280, 95 (1994).

${ }^{23}$ W. H. Matthaeus, S. Ghosh, S. Oughton, and D. A. Roberts, J. Geophys. Res. 101, 7619 (1996).

${ }^{24}$ J. Vestuto, E. Ostriker, and J. Stone, Astrophys. J. 590, 858 (2003).

${ }^{25}$ J. Belcher and L. Davis, J. Geophys. Res. 76, 3534 (1971).

${ }^{26}$ A. Barnes, in Solar System Plasma Physics, edited by E. N. Parker, C. F. Kennel, and L. J. Lanzerotti (North-Holland, Amsterdam, 1979), Vol. I, p. 251.

${ }^{27}$ L. W. Klein, D. A. Roberts, and M. L. Goldstein, J. Geophys. Res. 96, 3779 (1991).

${ }^{28}$ S. R. Spangler and L. G. Spitler, Phys. Plasmas 11, 1969 (2004). 